\title{
Urban growth and climate adaptation
}

\author{
Urban development induces local warming in addition to climate change. New research shows that urban growth, \\ climate change and urban adaptation interact nonlinearly and diurnally.
}

Lei Zhao

I eat stress is one of the most serious climate problems facing urban residents. The infamous Chicago heat wave in 1995, for example, caused more than 1,200 deaths $^{1,2}$. Absent measures to ameliorate it, heat stress in cities is expected to worsen in the coming decades due to rapid urban development coupled with climate change. Around $70 \%$ of the world's population is projected to live in cities by year $2050^{3}$. The potentially large socioeconomic impacts have motivated research into future urban microclimates under climate change and urban adaptation strategies. In this issue, E. Scott Krayenhoff and colleagues ${ }^{4}$ report that urban development and climate change interact nonlinearly, producing a combined warming effect that is less than their linear sum, and that infrastructure-based urban adaptation strategies bring marginal relief for projected night-time warming.

Conversion of undeveloped land to urban land (urban expansion) is known to generate local warming effects by altering biophysical properties of the land surface, adding to the non-local GHG-induced warming. Urban adaptation strategies seek to deliver local climate benefits by perturbing the urban surface energy balance. Previous studies modelling the effects of urban development, adaptation and/or climate change on urban temperatures have suggested unstable urbaninduced warming effects under climate change scenarios ${ }^{5,6}$ and varying effectiveness of adaptation strategies in bringing down daytime urban temperatures ${ }^{7}$, but results are inconsistent between studies and regions. In addition, most modelling studies assume a fixed urban representation over time, are based on shorter periods or lack a full diurnal evolution.

Using a set of regional climate simulations, Krayenhoff and colleagues ${ }^{4}$ provide decadal projections of the summertime urban temperature change in the contiguous United States by the end of the century induced by urban development, climate change, adaptation strategies and their dynamic interactions. They incorporate urban expansion dynamically into their simulations by introducing novel

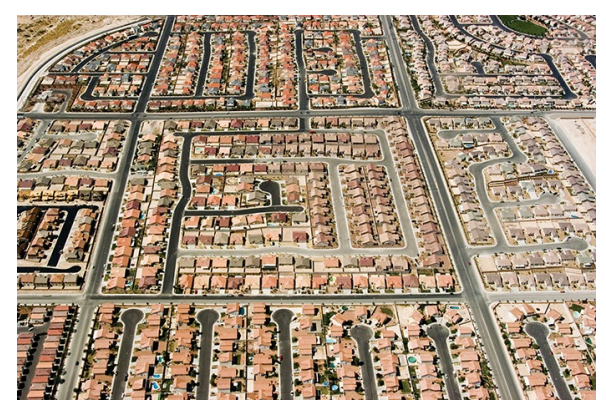

Credit: Lee Foster/Alamy Stock Photo

developments to the Weather Research and Forecasting regional climate model to allow subgrid representation of urban growth. Their results demonstrate significant afternoon and night-time urban warming induced by the interactive combination of urban growth and climate change by the end of the century. The urban warming effects are regional and diurnal, with pronounced afternoon warming in the humid eastern United States and strong night-time warming in the Appalachian, Great Lakes and California Central Valley regions. They further show that urban expansion and climate change interact nonlinearly, producing projected warming that is less than the sum of the two drivers across most of the contiguous United States during the evening and night-time. Interactive effects during the daytime, however, show less agreement between simulations. Despite a negative interaction between urban expansion and climate change, large projected urban warming still prevails across the contiguous United States, especially at night when urban development-induced warming is comparable to GHG warming.

These results raise the important question of what we can do. Krayenhoff et al. further examine the impact of commonly proposed urban adaptation strategies (street trees, cool and evaporative roofs) individually and collectively under climate change scenarios. They find that aggressive implementation of adaptation strategies may help reduce projected daytime urban warming and extreme heat (depending on the region and emissions scenario) but brings minimal relief at night. A strategy targeting nocturnal cooling by reducing thermal admittance shows moderate cooling ability at night. This raises serious concerns because high nighttime temperatures are more closely related to heat stress-induced mortality ${ }^{8}$. Importantly, the study argues that a 'full urban adaptation' to climate change based on infrastructurerelated schemes is implausible and hence reaffirms the crucial role of mitigation of GHG emissions for urban climate benefits.

Future research, and engineering and planning efforts to devise efficacious nighttime urban adaptation strategies, is needed. However, state-of-the-art regional and global climate models are still relatively primitive in representing cities and parameterizing urban physical and hydrological processes, which makes it even harder to model new urban adaptation strategies. The assessment of the diurnal interaction between urban development, climate change and urban adaptation provided by Krayenhoff and colleagues $^{4}$ underscores a need to consider the interactive effects between local (urban development and adaptation, for example) and non-local (GHG warming) drivers to correctly predict future urban climates and possibilities for urban adaptation.

\section{Lei Zhao}

Department of Civil and Environmental Engineering, University of Illinois at Urbana-Champaign,

Urbana, IL, USA.

e-mail: leizhao@illinois.edu

Published online: 12 November 2018 https://doi.org/10.1038/s41558-018-0348-x

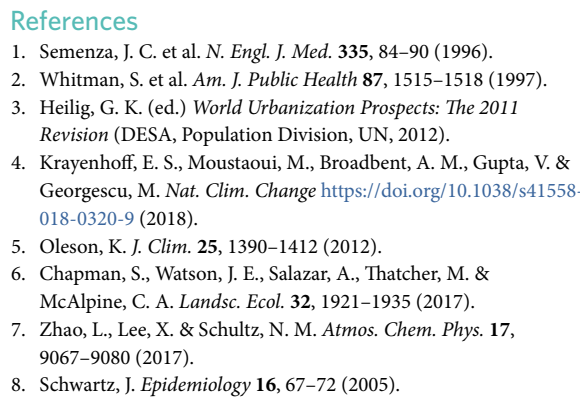

